\title{
La Discrepancia De Género En La Aptitud Intrínseca De Las Matemáticas
}

\author{
Luisa Morales-Maure, (Maestría) \\ Universidad de Panamá/ Departamento de Matemática \\ Universidad Especializada de las Américas, Decanato de Investigación. \\ Orlando García-Marimón, (Maestría) \\ Universidad de Panamá/ Departamento de Matemática \\ Raúl Montenegro, (estudiante) \\ Universidad Especializada de las Américas, \\ Facultad de Educación Social y Desarrollo Humano.
}

\section{doi: 10.19044/esj.2016.v13n2p331 URL:http://dx.doi.org/10.19044/esj.2016.v13n2p331}

\begin{abstract}
So far, studies have been conducted to assess the gender gap with respect to mathematics, being a hotly debated topic. This study contradicts previous works concluding that women are on average as good at math as men but are not able to Excel intellectually in this science for what we want to know what are the intrinsic skills that influence the perspective that have students toward mathematics. With base to the response of 500 students, men and women of the universities Panamanian. The results of our research show the differences that exist between men and women in the performance of mathematics, because males are more interested towards mathematical processes that women as their performance for them during school, while females are more mechanical reasoning to solve quickly and accurately presented tasks.
\end{abstract}

Keywords: Discrepancy, gender, aptitude, cognitive, perception, mathematics, experiment.

\section{Resumen}

Hasta ahora se han realizado estudios para evaluar la diferencia de género con respecto a la matemática, siendo un tema muy debatido. Esta investigación contradice los trabajos previos que concluían que las mujeres son en promedio tan buenas en matemáticas como los hombres pero que no son capaces de sobresalir intelectualmente en esta ciencia por lo que nos interesa saber cuáles son las aptitudes intrínsecas que influyen en la perspectiva que tienen los estudiantes hacia la matemática. Con base a la 
respuesta de 500 estudiantes, hombres y mujeres de las universidades panameñas. Los resultados de nuestra investigación muestran las diferencias que existen entre los hombres y mujeres en el rendimiento de las matemáticas, debido que los varones se interesan más hacia los procesos matemáticos que las mujeres pues su desempeño para ellos se destaca durante la fase escolar, mientras las féminas son más de razonamiento mecánico a fin de solucionar con rapidez y exactitud tareas presentadas.

Palabras clave: Discrepancia, genero, aptitud, cognitivo, percepción, matemáticas, experimento.

\section{Introducción:}

Para muchos aprender esta materia abstracta, es algo muy difícil, ya que son muchas las variables que influyen en la enseñanza y el aprendizaje; algo muy complejo que puede crear en los estudiantes actitudes negativas hacia esta ciencia.

Cuántas veces hemos oído la frase "las chicas no son buenas en matemáticas”. Y cuantos tipos de estereotipos y su gran peso han marcado un camino que no era el que esperábamos. En el artículo Mujeres y matemática publicada en la Revista Números 21 puede leerse:

A pesar de que las chicas superen la etapa escolar con mayor rapidez y provecho que los chicos, están en claro atraso respecto a éstos en materias científicas y matemáticas. Esto se debe a las expectativas sociales y a las consiguientes creencias de las chicas y no a habilidades innatas, como han demostrado las investigaciones. A menudo las chicas comienzan a desinteresarse por los asuntos técnicos muy temprano y estos, a la larga, tienden a surtir un efecto negativo en su profesión anterior. En casa y en el colegio se debe luchar contra este proceso desde el principio. (Salvador, A., 1991, p. 58)

Es indudable que algunas áreas laborales se encuentran altamente segregadas por el sexo. Tradicionalmente se ha hablado de una diferencia en las actitudes hacia las matemáticas por parte de los hombres y mujeres, se habla de una diferencia cerebral entre estos y una diferencia de interés sobre esta materia.

Entonces después de examinar numerosos estudios realizados acerca del tema la pregunta de investigación es ¿existen dificultades en cuanto a las habilidades matemáticas dependiendo de género de la persona? Este trabajo logrará afirmar o rechazar la hipótesis que se tiene acerca de que existe una discrepancia de género en la aptitud intrínseca hacia las matemáticas. La primera es de establecer baremos estadísticos que expliquen el comportamiento de los niveles de pensamiento mecánico, verbal y abstracto 
en hombres y mujeres ingresantes a las universidades panameñas; para la segunda analizar la confiabilidad del instrumento de actitud hacia la matemática con las diferencias de género.

\section{Marco Teórico:}

Un tema que hoy en día causa mucha conmoción es el pensar quien es aplicado en matemática, o a quien le gusta más, y si esto involucra hombres y mujeres aumenta el nivel, desde tiempos remotos se piensa que el hombre es más aplicado en matemática mientras que las mujeres no, pero la pregunta es ¿por qué se piensa esto?, ¿existen dificultades en cuanto a las habilidades matemáticas dependiendo del género de las personas? En muchos países alrededor del mundo se han hecho estudios referentes a este tema, uno de los motivos que ha llevado a los investigadores a realizarlos es quizá la cantidad predominante de hombres que se inclinan por el área de matemáticas al llegar a una institución de estudios superiores, situación que sostiene EURYDICE (2010):

Es indispensable comprender los patrones de género que se dan en estas áreas para avanzar hacia la igualdad de oportunidades en el empleo. Según los datos estadísticos, uno de estos patrones consiste, por ejemplo, en la infrarrepresentación de las mujeres en las áreas de matemáticas, ciencia y tecnología en la educación superior.

Otro motivo es posiblemente la presencia de estereotipos en la sociedad que aseguran que las personas tienen cierto rendimiento en un área determinada dependiendo del sexo, Inda-Caro, Rodríguez y Peña (2006) dice que:

En un primer momento, se señaló que las chicas superaban a los chicos en todas las asignaturas y en todos los niveles. Sin embargo, estas conclusiones fueron matizadas con posterioridad. Así, se estableció que las chicas despuntaban en aquellas disciplinas tradicionalmente calificadas de femeninas como, por ejemplo, la lengua, las humanidades o los idiomas extranjeros; al tiempo que estaban equiparando sus logros con los chicos en aquellas asignaturas que se percibían como masculinas, tal era el caso de las matemáticas y de la ciencia.

De acuerdo a dicha afirmación, no solo existen asignaturas que se consideran propias de las mujeres, así como otras tantas que se dicen son particularmente masculinas, y que de ello depende el rendimiento del individuo; sino que de igual forma asegura que dichos estereotipos no son tan inflexibles en el caso particular de las matemáticas, idea que también sostiene las autoras anteriores al decir que: 
En los resultados referentes a las competencias lectora y matemática es de destacar que se repiten los estereotipos tradicionales, las chicas han obtenido un rendimiento superior a los chicos en la competencia lectora mientras que en la competencia matemática se han invertido los datos. Sin embargo, hay que señalar que mientras en la competencia lectora las diferencias con base en el género son bastante destacables, en la competencia matemática la distancia entre los chicos y las chicas se iguala.

Después de expresado todo lo anterior, es necesario mencionar que en una cantidad significativa de los estudios realizados ha quedado demostrado que en lo que a las diferencias de género en matemáticas se refiere, los resultados son bastante diferentes y muestran cambios a través del tiempo, tal como lo asegura EURYDICE (2010). Los resultados del estudio TIMSS, en lo que se refiere a las diferencias de género en matemáticas, son bastante heterogéneos, y muestran cambios de un ciclo a otro. Por otro lado, existen investigaciones centradas en el estudio del género en educación, algunos de los más recientes estudios encontrados son; Cáceres (2007). Hacia un cambio social: reestructuración de roles y modelos educativos en la construcción del género. Santos y otros (2000) El harén pedagógico. Perspectivas de Género en la organización escolar. Lagarde (2015).

A pesar de ello, la gran mayoría de todos estos estudios reflejan que son los hombres quienes tienden a sobresalir con más aptitudes para las matemáticas; esto lo sostiene Burges (2006). Las diferencias sexuales más consistentes en este campo se han encontrado en el "test escolástico de aptitud matemática” (Scholastic Aptitude Test) en el que la ventaja masculina es clara; Delgado y Prieto (1996). Al igual que ocurre con las tareas especiales, los hombres superan a las mujeres en tareas matemáticas a partir de los doce o trece años; Maccoby y Jacklin (1974). Los niños tienden a calificar su competencia matemática como más alta que las niñas de igual habilidad. Así como también a este sexo se le asocia especialmente con los símbolos y su manipulación o al razonamiento matemático, Burges (2006) afirma que "El cerebro masculino tiene más habilidad para la manipulación formal de los símbolos, campo muy relacionado con el razonamiento matemático"; ISTIC (s.f.) de igual modo dice que "Los lóbulos temporales se activan bilateralmente (PET) en varones con pruebas de razonamiento matemático, esta específica activación cortical no es observada en mujeres”.

También se afirma que el hecho de que las mujeres no sobresalen en esta área las hace menos capaces de desarrollarla; sin embargo, hay excepciones como la primera mujer que recibe una medalla Fields en el año 2014 por su valiosa contribución a la Matemática (profesora en la Universidad de Stanford), lo cual no se pensaba que alguna vez ocurriese. Aunque todavía se mantiene el fenómeno de superioridad masculina en las habilidades matemáticas, existen estudiosos que se han adentrado en el 
campo causal, llegando a conclusiones que señalan distintos factores sociales como responsables del mismo, Coronado, Sandoval y Torres sostienen que:

Las causas de que los hombres destaquen más que las mujeres en matemáticas (y en otras áreas del conocimiento) se deben a factores culturales, a desigualdades sociales que han vivido las mujeres a lo largo de la historia y a la falta de oportunidades para acceder a la misma educación que los hombres. (2012)

Por su parte Hyde y Mertz (2009) sostienen que las mujeres son tan buenas en matemáticas como los varones en todos los niveles de exigencia, si se les dan las mismas oportunidades, concluyó este estudio realizado en Estados Unidos, y que contradice otros trabajos que afirmaban que las mujeres eran "incapaces" de sobresalir en esta ciencia por "diferencias biológicas". Sin embargo, todavía se piensa que los hombres, más que las mujeres, necesitan las matemáticas para su vida adulta y para conseguir buenos trabajos.

Algunos investigadores interesados en este tema como Thomas (2000), Willis (1996), Parker, Rennie and Fraser (1995), entre otros, indicaron que esta actitud negativa de las mujeres hacia el aprendizaje de las matemáticas, a su vez, con frecuencia constituye un factor poderoso que ha contribuido a su baja implicación y menor éxito que los hombres en las disciplinas que impliquen el manejo de contenidos matemáticos. Otras conclusiones de estas investigaciones están que chicos y chicas, con los mismos resultados en test de matemáticas, tienen ideas muy diferentes acerca de su habilidad. Los chicos son más seguros de sí mismos que las chicas en lo que respecta a la competencia matemática (Preckel, Goetz, Pekrun, y Kleine, 2008).

En la mayoría de las investigaciones nos dicen que los hombres son los que se aplican más en matemáticas que las mujeres, más sin embargo no todo el tiempo esta hipótesis es cierta, ya que Forgasz (1995) realizó una revisión extensa para intentar contrastar la hipótesis de las supuestas diferencias de género a favor de los hombres en el aprendizaje de las matemáticas y sus datos, de estudiantes australianos, revelaron que los chicos consideran las matemáticas más difíciles que las chicas, necesitando de ayuda adicional. Además, las chicas se interesaban y apreciaban más las matemáticas que los chicos. Por tanto, estos resultados parecen desafiar la hipótesis de las matemáticas como un dominio masculino.

Otras investigaciones como realizadas por la Dra. Rosa María Farfán Márquez y María de los Ángeles Cabañas Canto, nos dice que han encontrado que en su mayoría, los factores relacionados con los desempeños de los estudiantes hombres y mujeres, y estos se derivan, de alguna manera, por las imposiciones y costumbres que rigen la sociedad donde se desarrollan, por lo que podemos decir que los desempeños de las mujeres 
están regidos por las conductas, creencias y tradiciones que se han suscitado en su entorno social, y las cuales tienen su origen en la construcción social de género, que se ha hecho a través de la historia de la humanidad, y la cual asigna a cada individuo un papel a desempeñar. Es por ello que en la investigación en curso estamos interesadas en determinar cuáles son aquellos factores socioculturales que se encuentran relacionados con los éxitos de las mujeres en el área de matemáticas.

Esta investigación se realiza bajo una población estudiantil panameña correspondiente a la Universidad Especializada de las Américas, estudiantes de las carreras de Optometría y Biomédica que son licenciaturas en la cual hay estudiantes de diferentes sexos, donde se buscara revisar los objetivos propuestos en este estudio.

\section{Justificación}

Una de las áreas más a menudo investigadas son las relacionadas con el género, en la educación, es decir, la comparación de las características y el rendimiento masculino y femenino. Maccoby y Jacklin (1974) concluyeron, que persisten algunos patrones, por ejemplo, la superioridad femenina en las habilidades verbales y la masculina en las habilidades matemáticas, es difícil de desenredar la influencia de los estereotipos sobre las percepciones y comportamiento hacía, eventos, objetos, y también para separar los comportamientos innatos o aprendidos en el desarrollo de las diferencias cognitivas.

William et al. (2000) sugiere asimismo que las diferencias sexuales en la cognición son pequeñas y se han estrechado aún más en algunos temas en los últimos años. Quizás el descubrimiento más importante de la literatura y análisis es que el sexo-diferencias en el rendimiento, incluso en materias como las matemáticas y la ciencia, son pequeñas y han ido disminuyendo constantemente durante los últimos 20 años. Algunas de las pruebas muestran una diferencia de medias estándar en favor de los chicos o chicas de más de 0,4 cuales significa que menos del 4\% de la variación en las puntuaciones de las pruebas de los individuos está relacionada con las diferencias de sexo

De hecho, a raíz de Maccoby y Jacklin (1974) y Hyde (2005) sostiene que los hombres y las mujeres son de hecho muy similar en la mayoría, aunque no todos, en la variable psicológica. Es por ello que encontrar una respuesta a la siguiente pregunta ¿existe dificultades en cuanto a las habilidades matemáticas dependiendo del genero de las personas? Ha sido uno de los motivos que ha llevado a realizar el siguiente estudio. En la medida que se investiguen las aptitudes de los estudiantes, se obtendrá información que nos permita aceptar o rechazar las hipótesis que se tiene; muchas son las personas que consideran que las matemáticas son para 
hombres y no para las mujeres, ya que consideran que simplemente, son ellos quienes se aplican más, pero nos deja la interrogante de ¿Por qué se piensa esto?, estudios internacionales, muestran que en matemática los niños y niñas tienen resultados similares en cuarto y octavo grado, la ventaja en los niños surge en los años escolares posteriores y es especialmente notable entre los estudiantes que asisten a programas de enseñanza universitaria.

Se identifican y analizan también los errores que cometen los estudiantes al trabajar con los temas que conforman el área de matemática en el nivel universitario. La discrepancia de género nos lleva a investigar cual son las diferencias entre hombres y mujeres en cuanto a las capacidades matemáticas dentro de su carrera universitaria. Estas discrepancias de género han logrado marcar una diferencia en el desempeño de muchas personas partiendo de distintos enfoques psicológicos.

Se estudian también las aptitudes, la autoconfianza y las emociones que despierta esta disciplina, y se profundiza desde distintas perspectivas, la problemática de géneros y matemáticas. Para esto hemos creado un instrumento que nos permita medir que existe dicha discrepancia.

\section{Método}

\section{Diseño de investigación}

Debido a las características de la muestra y al problema de la investigación, se trata de un estudio descriptivo -en el que se caracterizan las actitudes- y correlacional -con el propósito de medir el grado de relación que existe entre las variables del estudio- (Hernández, Fernández y Baptista, 2010).

\section{Tipo de estudio:}

Esta investigación es descriptiva, en razón de que se sustentó en la aplicación de un cuestionario, con preguntas relativas para reconocer la percepción hacia la Matemática, y su influencia en el rendimiento académico con estudiantes universitarios. La investigación trata de determinar la fuerza de asociación o correlación entre variables, la objetivación de los resultados a través de una muestra para hacer inferencia a una población de la cual toda muestra procede.

\section{Objetivos}

El objetivo de este trabajo es determinar si existen evidencias que sustenten la discrepancia en matemática, de efectos interactivos de la variable género, en relación con los desempeños en matemática de alumnos en las carreras de Biomédica y Optometría de la Universidad Especializada de las Américas. Este objetivo puede ser desglosado en las siguientes preguntas de investigación: 
- ¿Es significativa la distancia entre el promedio de calificaciones esperado para hombres y mujeres en los resultados de las pruebas de matemática ("efecto género")?

- ¿La percepción matemática por género es significativo? ¿Existen diferencias en la variación de los rendimientos de acuerdo con el género?

Dado que se especificarán modelos bivariados, en cada caso se podrán comparar directamente los resultados de matemática y estimar sus covarianzas.

\section{Población y Muestra}

La población $(\mathrm{N})$ que participó en este estudio estuvo conformado por estudiantes de la Universidad especializada de las Américas UDELAS. Los grupos estudio serán seleccionados de las carreras de Biomédica y Optometría, que a su vez fueron seleccionados por el semestre (ambas carreras) y la Extensión Regionales (n1); los estudiantes (n) fueron elegidos probabilísticamente al azar para evaluar el rendimiento de acuerdo al género en matemática, se describen en la siguiente tabla:

Tabla 1: Distribución por género y carrera profesional de la muestra del estudio

\begin{tabular}{cccc}
\hline Facultad y Carrera & Total & Hombres & Mujeres \\
\hline Facultad de Biociencias y Salud Pública & 847 & datos & datos \\
Ingeniería en Biomédica & $\mathbf{1 9 7}$ & $\mathbf{1 2 3}$ & $\mathbf{7 4}$ \\
Facultad de Ciencias Médicas y Clínicas & 736 & datos & datos \\
Doctorado Profesional en Optometría & $\mathbf{1 7 1}$ & $\mathbf{3 3}$ & $\mathbf{1 3 8}$ \\
\hline
\end{tabular}

\section{Instrumento}

El instrumento utilizado para la recolección de datos fue la encuesta que nos permitió conocer por medio de resultados la discrepancia de género en las aptitudes hacia las matemáticas.

Para elaborar nuestra encuesta las dividimos en tres tipos de variables, las cognitivas, las afectivas y las conductuales, las cognitivas son para saber más sobre los conocimientos que responde a la encuesta, de esta manera se tendría una idea como está la persona en cuanto a sus conocimientos en las matemáticas. Las afectivas tienen la finalidad de poder distinguir cual es el sentir de la persona, ya sea miedos, angustias, nerviosismo, etc. Y no menos importante se encuentran las conductuales las cuales nos permiten conocer el comportamiento de una persona frente a una situación en particular. Inicialmente a la hora de laborar nuestra encuesta se tuvieron ciertos inconvenientes debido a que algunas de las preguntas no permitían que el encuestado respondiera con claridad y tampoco permitía evaluar si realmente la hipótesis sobre la discrepancia de género en las matemáticas existe. La encuesta realizada posee cinco opciones las cuales se clasifican en: 
- Opción 1 Totalmente de acuerdo, es para aquellas personas que se encuentran completamente a favor de algún ítem.

- Opción 2 De acuerdo, es para aquellas personas que se encuentran a favor del ítem, sin embargo, no están muy convencidas.

- Opción 3 Indeciso/ Indecisa, es para aquellas personas que no saben si están a favor o en contra del ítem afirmado.

- Opción 4 En desacuerdo, es para aquellos que están en contra de la afirmación realizada.

- Opción 5 Totalmente en desacuerdo, es para aquellos que rechazan totalmente la afirmación del ítem.

Se elaboró una encuesta masculina y una femenina las cuales se dividieron en partes equitativa. A la hora de tabular los datos se tomaron dos opciones, el número (1) para identificar el género femenino y el número (2) el género masculino.

\section{Procedimiento de recogida y análisis de datos}

A partir de la revisión de las fuentes bibliográficas relacionadas con el objeto de estudio y del análisis previo de los ítems de otros cuestionarios (EURYDICE, 2010; Inda-Caro, Rodríguez y Peña, 2006; Burges, 2006), se seleccionaron textualmente algunos ítems, mientras que otros fueron creados, se adaptaron y recibieron modificaciones. A continuación, se procedió a la organización y estructuración de los ítems en componentes buscando su correspondencia con los tres componentes de las actitudes: cognitivo, afectivo y comportamental o conductual. Se procedió a aplicar el cuestionario a un número reducido de estudiantes con la finalidad de validarlo y evaluar su confiabilidad para detectar cualquier posible error en su estructura y contenido. Para esta investigación se obtuvo un índice de confiabilidad del 0.872 , considerándose así la encuesta válida y confiable.

Una vez efectuada la aplicación de la escala de percepción por género hacia la matemática que nos ha permitido recopilar, vaciar y codificar los datos de las 368 respuestas obtenidas, se procede a presentar los análisis realizados con la finalidad de extraer los resultados pertinentes de acuerdo con el objetivo que nos habíamos propuesto.

\section{Resultados}

Se presenta en primer lugar el análisis descriptivo; con ello se ha caracterizado la muestra según las diferentes variables estructurales. Se han 
utilizado distintas herramientas para resumir la información que contiene la muestra: tablas, gráficos, medidas de tendencia centra y de dispersión.

En la siguiente tabla se presenta la percepción tanto femenina como masculina con respecto a las opciones de respuesta en los ítems; el cual el ítem 1 simbolizaba totalmente en desacuerdo, el 2 en desacuerdo, el 3 indeciso, el 4 de acuerdo y el 5 totalmente de acuerdo, se muestra un mayor incremento en la percepción masculina en el ítem 2.

Tabla 2: Distribución de frecuencia por género de la percepción hacia la matemática en la muestra del estudio

\begin{tabular}{ccccccc}
\hline Descripción & $\mathbf{1}$ & $\mathbf{2}$ & $\mathbf{3}$ & $\mathbf{4}$ & $\mathbf{5}$ & Total \\
\hline Percepción General & $\mathbf{1 5}$ & $\mathbf{2 3 2}$ & $\mathbf{9 2}$ & $\mathbf{2 9}$ & $\mathbf{0}$ & $\mathbf{3 6 8}$ \\
Percepción Femenina & 13 & 97 & 84 & 18 & 0 & 212 \\
Percepción masculina & 4 & 122 & 18 & 12 & 0 & 156 \\
\hline
\end{tabular}

La percepción de la aptitud de hacia las matemáticas atendiendo a los efectos observados, se encontró la coincidencia entre ambas muestras en que las mujeres, respecto de los hombres, muestran una mayor falta de confianza en sus logros futuros en el área de las matemáticas. Informan de un mayor pensamiento estereotipado (sosteniendo en mayor medida que las matemáticas son cosa de hombres), menor competencia percibida para el aprendizaje de las matemáticas, menor ansiedad ante las matemáticas y menor atribución del éxito a causas externas. Calero, Sánchez y Núñez (2015) analizaron las diferencias por género y edad, encontrando que los hombres activos mostraban mayores niveles de interés y disfrute, así como mayor competencia en su participación en las actividades físicas que las mujeres.

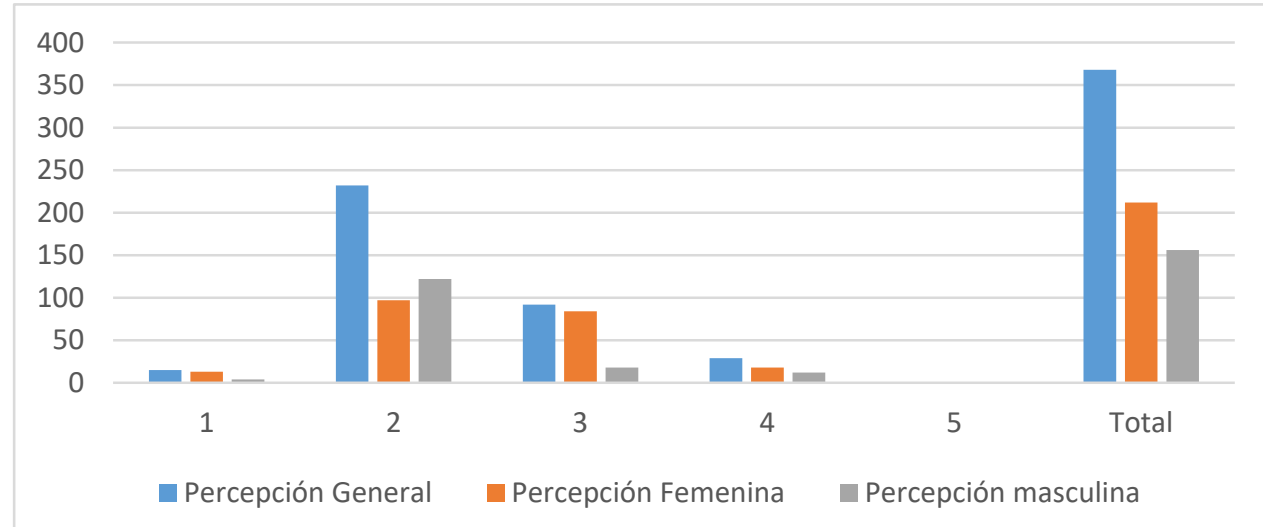

Figura 1. Distribución de la frecuencia de la percepción hacia la matemática general y por género.

En la Figura 1 se ven los datos presentados anteriormente, pero esta vez en gráfico de barras, en el cual está la distribución del sexo (masculino, 
femenino) con respecto a los ítems. En la misma se observa que existe una baja percepción negativa del sexo masculino correspondiente a estos ítems y un incremento de la percepción femenina en el ítem 3, el cual tienden a estar más indecisas que los hombres.

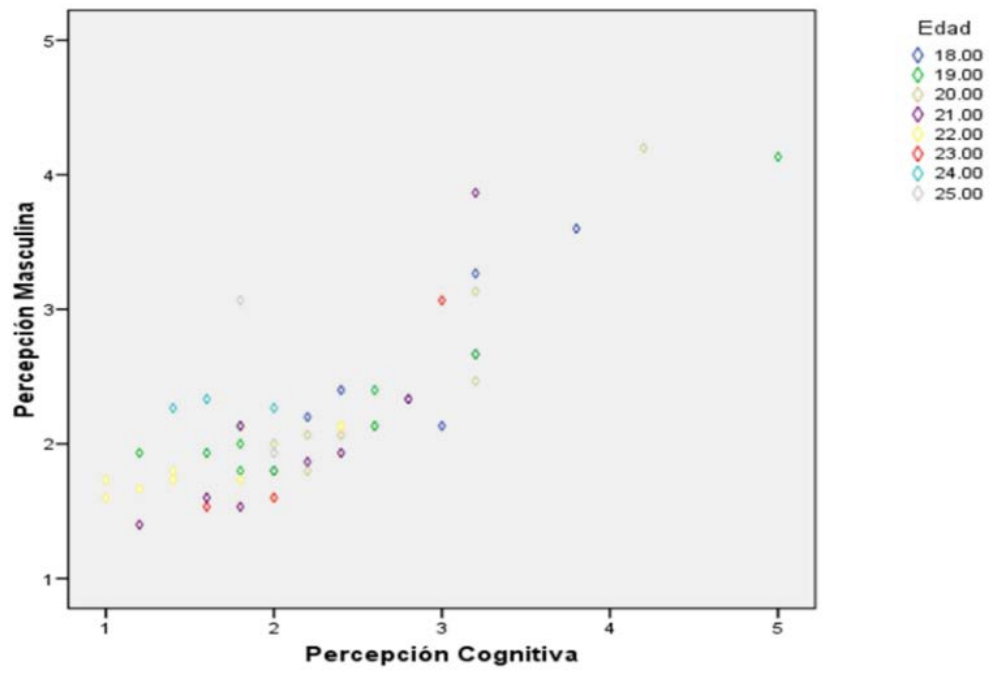

Figura 2. Diagrama de dispersión de la percepción matemática cognitiva y la percepción masculina en los estudiantes de la muestra.

Respecto a la figura 2, a los hombres no se les hace difícil aprender matemáticas y cuentan con más habilidades e interés. En contraste las mujeres cuentan con actitudes negativas para toda comprensión con la simple presencia de los símbolos matemáticos y números, actitud que bloquea sus posteriores adquisiciones. En este diagrama nos muestra una correlación de $0.752^{* *}, \rho=0.00$, resaltando que la percepción masculina es negativa ($0.258^{* *}, \rho=0.00$, correlación débil) esto implica que, a mayor edad, hay cierta percepción negativa sin importar el género. Esto lo sustentamos con el trabajo de Alonso, Sáez y Picos (2004) dado que cuanto mayor es esta percepción de competencias Matemáticas, más probabilidad hay de que se trate de un alumno con gusto por las Matemáticas. Estos autores que la percepción disminuye de manera progresiva al aumentar la edad de los alumnos. Tal es así que si en $3^{\circ}$ de primaria el $50 \%$ de los alumnos se consideran buenos para las Matemáticas, cuando llegamos al primer curso universitario esta cifra ha disminuido hasta el $4 \%$.

La Figura 3 se presenta la existencia una percepción masculina negativa en edades jóvenes, lo que implica que en la población masculina juvenil hay una diferencia de género con la población femenina respecto a la matemática, ya que si observamos el diagrama nos muestra que los datos tienden a estar más acumulados en edades jóvenes. 


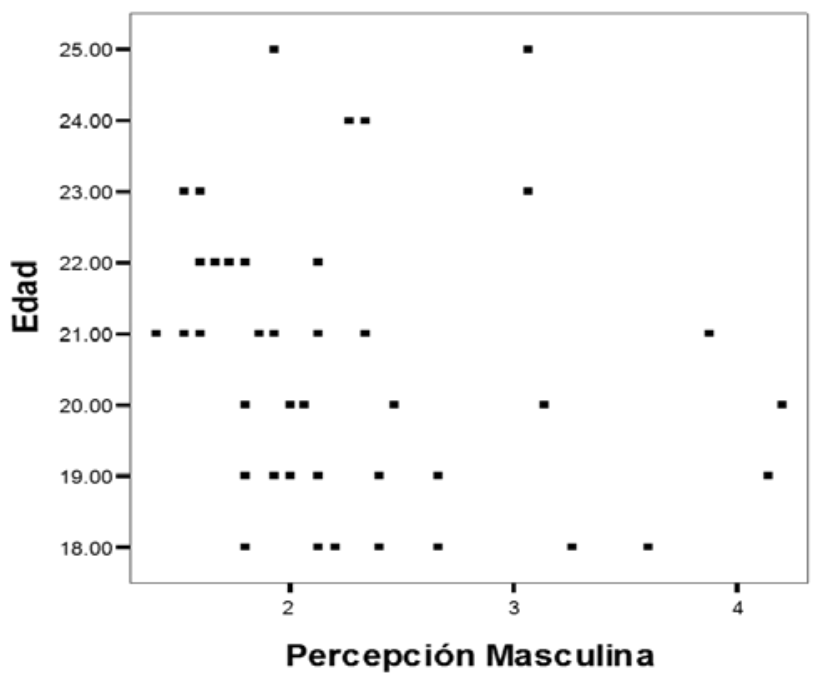

Figura 3. Diagrama de dispersión de la percepción masculina con respecto a la edad.

Por otro lado, la Figura 4 se muestra la percepción cognitiva con respecto al género femenino, y se muestra que también al igual que los hombres existe una percepción negativa solo que esta sería mucho menos, y se muestra que los datos tienden a acumularse en el ítem 3. Esta opción 3 que corresponde a indeciso es la más seleccionada, indicando que hay una inseguridad de parte del género femenino con respecto a la materia. Existe algunos estudios que indican que los niños reportan un mayor éxito en comparación con las niñas acerca de su desempeño en matemáticas, ciencias y tareas relacionadas con computación (Drundell y Haag, 2002; Pajares, 1996; Schunk y Pajares, 1991; Vekiri y Chronaki, 2008; Zimmerman y Martinez-Pons, 1990), a pesar de que las niñas tienen un rendimiento académico tan bueno como los niños (Betz y Hackett, 1983).

Sin embargo, cuando se trata de tareas como leer o escribir, las niñas reportan una mayor autoeficacia que los niños (Pajares \& Valiante, 2001). 

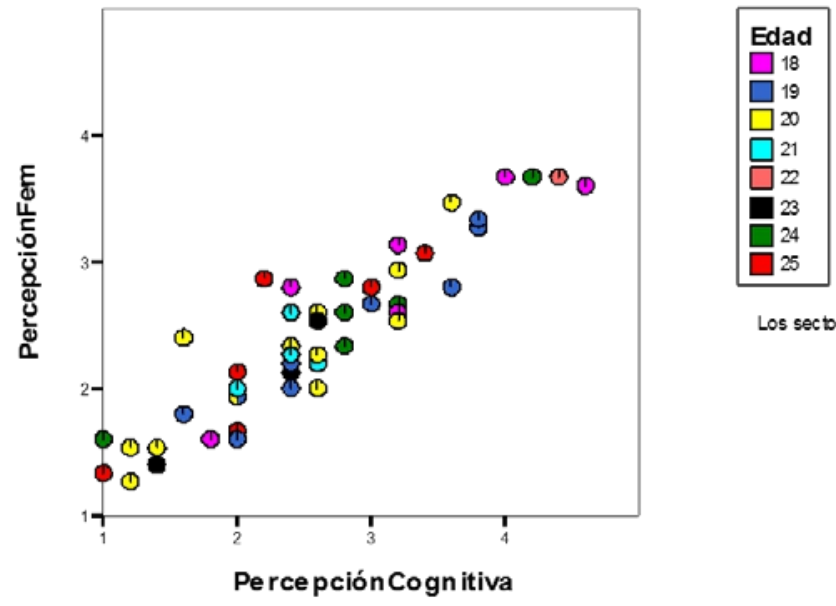

Los sectores muestran frecuencias

Figura 4. Diagrama de dispersión de la percepción cognitiva con respecto a la percepción femenina

Tan solo escuchar: "Las matemáticas" resulta para algunos estudiantes una materia en su concepto difícil, aburrida y sin importancia; la cual se han llevado como un mal hábito en la sociedad donde se desarrollan. Estereotipando por género quienes pueden lograr avanzar y quienes desertan de la misma. Al respecto, Bandura (1997) señala que mientras que los niños tienden a "inflar" su sentido de competencia, las niñas menosprecian sus capacidades, lo anterior debido a que ambos aprenden a valorar sus capacidades con base en lo que sus familias les enseñan, y esto generalmente se encuentra sesgado por los estereotipos de género.

Los resultados indican que una visión más estereotipada acerca de hombres y mujeres se relaciona en ambos casos con falta de autoeficacia. Esto es congruente con la teoría de Bem (1974) acerca de que las personas más estereotipadas son más rígidas cognitivamente hablando y experimentan un mayor malestar psicológico al desempeñar tareas no tradicionales, lo cual tiene implicaciones en su salud en el sentido de que les causa mayor estrés tener que enfrentarse a situaciones nuevas o situaciones que no sean congruentes con sus estereotipos. 


\section{Conclusión:}

Durante el proceso de nuestra investigación se muestran las diferencias que existen entre los hombres y mujeres en el rendimiento de las matemáticas. Debido que los varones se interesan más hacia las matemáticas que las mujeres; pues el desempeño de ellos se destaca durante la fase escolar, carrera universitaria y vida cotidiana. Se logra observar a la hora de aplicar las encuestas que muchos varones aceptaban realizarlas obteniendo cooperación por parte de ellos; en cambio las mujeres no le daban la importancia o el tiempo, y las que decidieron llenar las encuestas mostraban un sentido de personalidad apática porque calificaban que su lado femenino sabia más que el varonil.

Los resultados reflejaron que conforme el hombre aumenta su edad tiende a pensar que se aplica más en matemática que las mujeres y también que existe un nivel considerable en indecisión en ellas. Las matemáticas siempre serán un papel importante dentro de la educación estudiantil para el día de hoy y el de mañana en nuestras futuras generaciones solo deben confiar en sus propias habilidades y desempeño de aprendizaje dentro y fuera del aula de clases. Por otro lado, dependerá el tipo de enseñanza por parte de los maestros y padres al inculcar que las matemáticas son una actividad apropiada para cualquier género, así no se fomentara que solo es apropiada para los varones y se pueda lograr un cambio en disminuir los bajos rendimientos en las actividades relacionadas con el tema de las matemáticas la utilidad de estas y las relaciones con su educación futura u otras actividades también atribuyen gran valor para una mejor educación en nuestro país. Las matemáticas son y serán una fuente de aprendizaje y de enseñanza para hombres y mujeres en nuestra educación básica como parte del proceso de transmisión cultural.

La investigación sobre las capacidades cognitivas de los machos y hembras, desde el nacimiento hasta la madurez, no apoya la afirmación de que los hombres tienen mayor aptitud intrínseca de las matemáticas. Los varones y mujeres no difieren en lo cognitivo habilidades en los fundamentos de la matemática y científica pensando; tienen habilidades comunes para representar y aprender acerca de los objetos, números, el lenguaje y el espacio. Hombre y mujer aprovechan estas capacidades de la misma manera, por lo la misma hora, para dominar los conceptos y las operaciones de matemáticas elementales. A pesar de que los niños y niñas de más edad mostrar algo diferentes perfiles cognitivos, las diferencias son complejos y sutiles (que no es el caso, por ejemplo, que las mujeres son verbales y los hombres son espaciales). Estas diferencias tienden a sean pequeños, y ellos se derivan principalmente de las diferentes estrategias. 


\section{References:}

1. Alonso, S. H., Sáez, A. M., y Picos, A. P. (2004). ¿Por qué se rechazan las matemáticas? Análisis evolutivo y multivariante de actitudes relevantes hacia las matemáticas. Revista de Educación, (334), 75-95.

2. Bandura, A. (1997). Self-efficacy the exercise of control. New York: W.H. Freeman and company.

3. Bem, S. L. (1974). The measurement of psychological androgyny. Journal of consulting and clinical psychology, 42(2), 155-162.

4. Betz, N. E. y Hackett, C. (1983). The relationship of mathematics self-efficacy expectations to the selection of science-based college majors. Journal of Vocational Behavior, 23, 329-345.

5. Burges, Lucrecia. (2006). Diferencias mentales entre los sexos: Innato versus adquirido bajo un enfoque evolutivo. Ludus Vitalis, XIV (25), 43-73.

6. Cáceres, P. (2007). Hacia un cambio social: reestructuración de roles y modelos educativos en la construcción del género. En Lorenzo y otros. Gestionando los Nuevos Actores y Escenarios de la Formación en la Sociedad del Conocimiento. Granada: Adhara.

7. Calero, I. A., Sánchez, A. C., y Núñez, J. A. L. (2015). Análisis de las actividades extraescolares en función de la variable género en el alumnado de primaria de la provincia de granada. European Scientific Journal, 11(13).

8. Coronado, S., Sandoval, S., \& Torres, A. (2012). Diferencias de género, factores que inciden en el rendimiento matemático de licenciaturas económico administrativas. Sinéctica, (39), 01-22.

9. Delgado, A. R. y Prieto, G. (1996). Sex differences in visuospatial ability: do performance factors play such an important role? Memory \& Cognition, 24(4), 504-510.

10. Durndell, A. y Haag, Z. (2002). Computer self-efficacy, computer anxiety, attitudes towards the internet and reported experience with the internet, by gender, in an East European sample. Computers in Human Behavior, 18(5), 521-535.

11. EURYDICE. (2010). Diferencias de género en los resultados educativos: medidas adoptadas y situación actual en Europa. Ministerio de Educación. ISBN 978-92-9201-131-4. http://www.educacion.es/cide/eurydice

12. Forgasz, H., (1995). Gender and the relationship between affective beliefs and perception of grade 7 mathematics classroom learning environments. Educational Studies in Mathematics.28(3), 219-239. 
13. Hernández, R., Fernández, C., \& Baptista, P. (2010). Recolección de datos. Metodología de la investigación. México: Mac Graw Hill, 342482.

14. Hyde, J. S., y Mertz, J. E. (2009). Gender, culture, and mathematics performance. Proceedings of the National Academy of Sciences, 106(22), 8801-8807.

15. Hyde, J.S. (2005). The gender similarities hypothesis. American Psychologist, 60(6), pp. 581-592.

16. Inda-Caro, M., Rodríguez, M. C. \& Peña, J. V. (2010). PISA 2006: la influencia del género en los conocimientos $\mathrm{y}$ competencias científicas. Revista Iberoamericana de Educación, 51, 1-12.

17. ISTIC. (s.f.) Cerebro de hombre, cerebro de mujer. Recuperado 24 de julio de 2016. http://cettenerife.org/descargas/cerebro_de_hombre_y_mujer_2.pdf

18. Lagarde, M. (2015). Identidad de género y derechos humanos. La construcción de las humanas. Disponible desde la URL: http://www.catedradh.unesco.unam.mx/

19. Maccoby, E.E. y Jacklin, C.N. (1974). The Psychology of sex differences. Stanford: Stanford University Press.

20. Marquez, R. M. F., \& Canto, M. D. L. A. C. Diferencias de género en matemáticas, un problema sociocultural.

21. Pajares, F. y Valiante, G. (2001a). Influence of self-efficacy on elementary students writing. Journal of educational research, 90(6), 353-360.

22. Parker, L. H., Rennie, L., y Fraser, B. (Eds.). (1995). Gender, science and mathematics: Shortening the shadow (Vol. 2). Springer Science \& Business Media.

23. Preckel, F., Goetz, T., Pekrun, R., \& Kleine, M. (2008). Gender differences in gifted and average-ability students comparing girls' and boys' achievement, self-concept, interest, and motivation in mathematics. Gifted Child Quarterly,52(2), 146-159.

24. Salvador, A.; Salvador, A.; Molero, M. (1991). Mujeres y Matematicas: un estudio diferencial. Números, 21, pp. 57-65. Tenerife.

25. Santos Guerra, M.A. y otros. (2000). El harén pedagógico. Perspectiva de género en la organización escola. Barcelona: Graó.

26. Schunk, D. H., \& Pajares, F. (1991). The development of academic self-efficacy. En A. Wigfield, y J.S. Eccles (Eds.), Development of achievement motivation (pp. 16-31), New York: Academic Press.

27. Thomas, J. P. (2000). Influences on mathematics learning and attitudes among African American high school students. Journal of Negro Education, 165-183. 
28. Vekiri, I. y Chronaki, A. (2008). Gender issues in technology use: perceived social support, computer self-efficacy and value beliefs, and computer use beyond school. Computers y Education, 51(3), 1392-1404.

29. Wiliam, D., Warren, S. W., Cole, N. S., Hakel, M. D., Torrance, H., \& Pryor, J. (2000). Assessment: social justice and social consequences.

30. Willis, S. (1996). Gender justice and the mathematics curriculum: Four perspectives. In Gender, science and mathematics (pp. 41-51). Springer Netherlands.

31. Zimmerman, B. J. y Martinez-Pons, M. (1990). Student differences in self-regulates learning: relating grade, sex and giftedness to selfefficacy and strategy use. Journal of Educational Psychology, 82(1), 51-59. 